\title{
Generation of anisotropic networks for simulations of signal spreading in excitable cell systems
}

\author{
Petr Vápenka and Igor Schreiber
}

Manuscript received on July 14, 2012 / accepted on November 6, 2012

\begin{abstract}
We describe a technique of constructing an anisotropic lattice of coupled subunits and apply it to assemblages of excitable myocardial cells conducting an electric impulse. The cells are assumed arranged in a planar network comprising a few thousands of cells, each having at most eight connections to neighbors. The connections are characterized as being one of four kinds: horizontal, vertical, diagonal and inversely diagonal. The connectivity in the cellular network is constructed randomly with prescribed fractions of each of the four kinds of connections. These fractions are taken from literature and characterize certain types of cardiac tissues, the left ventricle in particular. The nodes in the network correspond to cells, which are treated as point objects described by ordinary differential equations. For the model of the left ventricle we study spreading of the action potential initiated by an impulse stimulus of a small cluster of cells.
\end{abstract}

Keywords: cellular network, excitability, action potential, cardiac waves.

\section{INTRODUCTION}

Biological systems may be often treated as subsystems interacting with each other, creating thereby a compound unit displaying collective dynamics that cannot be displayed by a single subunit. The most conspicuous example is a tissue made up of cells. The interactions are based largely on the mass and charge exchange. Generally, the system includes subunits/cells with complex chemical kinetics interacting via electro-diffusive fluxes. The system may be seen as a network or a lattice possessing certain connectivity of its nodes. The network may have a regular connectivity, but a more appropriate view is to include local irregularities while maintaining an overall characteristic structure. In this work we apply this idea on cardiac tissue. This, along with others, such as nerve tissue [1] (predominantly composed of neurons and glial cells), liver (consisting of hepatocytes), oocytes of the frog
Xenopus [2] or pancreatic insulin secreting beta-cells [3] is a group of cells that are characterized by excitability or oscillatory behavior. These properties are the key for many physiological functions. Oscillations of $\mathrm{Ca}^{2+}$ ions in hepatocytes are essential for their proper function [4], likewise repetitive increase in calcium levels in muscle cells initiate contraction of muscle fibers [1].

Many mammalian cells contain gap junctions, which are channels that connect the cytosol of neighboring cells. Gap junctions enable them to communicate and transmit signals via second messengers. The channels themselves are complex structures with complex dynamics. In cardiac muscle, gap junctions mediate transfer of action potential coupled with mechanical contraction. Dynamics of the heart may include various pathological conditions such as different types of arrhythmias or ischemia. Such disorders can be accompanied by a reduced number of gap junctions [5]. It is therefore of great interest to understand the 
role of gap junctional coupling in cellular networks and its effect on the formation of these states. Previous work elucidated some aspects of dynamics of wave patterns in cellular networks and effects of irregularities and varying coupling strength. Stability of planar waves in two-dimensional (2D) arrangement of excitable cells with nonuniform distribution of coupling strengths was examined for two regular topologies of cells having four or six neighbors [6]. Spiral wave break up in a rectangular array of excitable cells with four coupled neighbors and its persistence in the array with incomplete connectivity [7] has been numerically studied. Effect of spatial heterogeneities in a layer of coupled chicken heart cells on spiral wave generation and break up was studied experimentally and modeled using a cellular automaton model on a 2D lattice [8]. Role of extraneous perturbations on spiral wave formation was modeled in a $2 \mathrm{D}$ grid of cells with eight coupled neighbors $[9,10]$.

This paper attempts to further clarify the issue of anisotropy/ coupling relations by constructing a class of networks with connectivity corresponding to the heart tissue and examining basic dynamical properties of spreading excitation waves. To achieve this goal, we use existing software systems for construction of networks based on Python programming environment [11, 12], build an extension allowing us to generate specific types of networks, and develop a programme translating the network into a software for stability and bifurcation analysis and dynamical simulations [13].

\section{NETWORK OF HEART CELLS}

Contraction of heart cells - myocytes - is a complex process that is initiated by depolarization of the cytoplasmic the membrane. This depolarization is generated in the right atrium, where a bundle of specialized cells called the sino-atrial $(S A)$ node is located. These cells spontaneously oscillate, i.e., there is periodic cytoplasmic membrane depolarization/repolarization. At the resting state there is a constant membrane depolarization. Upon external stimulation exceeding a threshold voltage, action potential (AP) is generated which is transmitted to neighboring cells. Then the membrane repolarizes and the whole process is repeated. The sinoatrial node impulse spreads through the atrial contractile myocytes to the atrio-ventricular (AV) node, which provides a significant delay in the action potential (approximately $0.1 \mathrm{~s}$ ). This allows the chamber to fill the entire volume of blood before ventricles are excited and contracted. AP is then spreading through the Hiss bundle, which is the only place that electrically connects atrium and ventricle. It is therefore a crucial element in the trans- mission of the AP to the ventricles. Hiss bundle extends through the septum (wall between the ventricles) and is split into left and right parts, containing very elongated cells called Purkinje fibers. These are linked to the myocytes in ventricles. These cells are specialized in rapid transmission of the AP.

There are many types of myocytes in the heart. Of interest here are contractile myocytes, which are mostly longitudinal cells with one nucleus. The cells are strongly mutually linked both mechanically and chemically. Areas in which the cells connect in the longitudinal direction are called intercalated disks. They are located at the longitudinal ends of the myocytes and clearly define their boundaries [16]. In these areas there are different kinds of intercellular connections. Desmosomes provide mechanical connections of myocytes. For this work, the most important connections are gap junctions (GJ) - the regions containing aqueous pores that connect the cytosol of adjacent cells. GJs allow cells to communicate chemically and electrically by allowing ions, metabolites and other small molecules (up to about $1000 \mathrm{Da}$ [14]) to diffuse. They also allow for transmission of the action potential [15].

Within the myocyte itself, the AP is spreading along its membrane, which is then said to be excitable. Transport through the membrane is mediated by two main mechanisms. The first of them is an active transport, which uses energy (mostly in the form of ATP) to transport particles along the electro-chemical gradient. The second one is a passive transport, which takes place spontaneously against electro-chemical gradient. The passive transport is often facilitated by specific channels. At steady state the membrane has certain nonzero voltage. In electrophysiology, it is customary to measure the potential within cells with respect to the external environment, which is assigned zero potential. According to this convention the membrane has a negative resting voltage. An increase of voltage (potential increase in the cytosol) is known as depolarization. The process by which the voltage decreases after membrane depolarization is called repolarization. Hyperpolarization occurs when the voltage drops below the resting level. Sodium or calcium ions incoming through specific channels are the major cause of the depolarization. The same effect has the flow of negatively charged chloride anions that are transported from the cytosol into the extracellular environment. Other pumps and channels are involved, in particular $\mathrm{Ca}^{2+}$ channels of L-type. Resting voltage of cells in the heart is about -80 to $-90 \mathrm{mV}$. During depolarization the voltage rises to $+30 \mathrm{mV}$. Most of the channels are voltage gated [17].

Figure 1 shows a typical arrangement of myocytes in the tissue. The myocytes may be branched, which allows for connections to other cells in various spatial directions. Although majority 


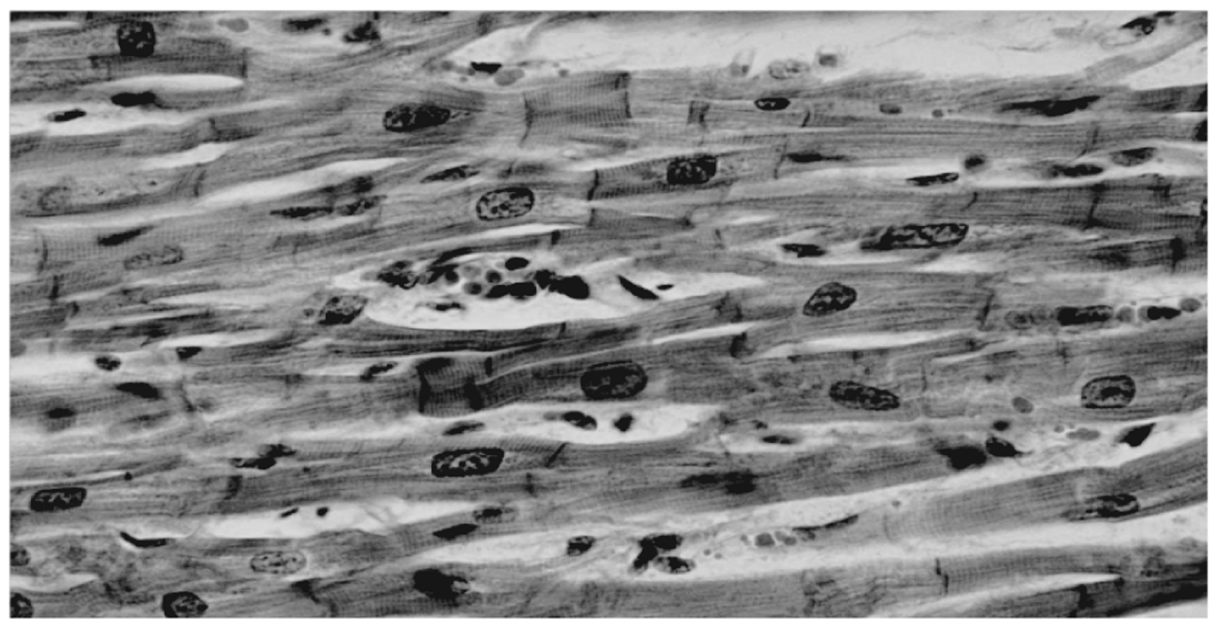

Figure 1 - An example of cardiac muscle tissue. Myocytes are oriented horizontally, rounded shapes are nuclei, short vertical bars are intercalated disks, branching of individual cells occurs throughout the region (modified from [17]).

of gap junctions occur in intercalated disks, the cells are coupled via GJ to all surrounding cells even though not to the same extent. Thus the network is anisotropic. Our model of the network attempts to capture the outlined features.

\section{MODEL OF THE AP IN A SINGLE CELL}

The primary model to describe the action potential in an isolated myocyte is based on the theory of Hodgkin \& Huxley for nerve cells [18]. The model can be schematically written as an electrical circuit, where the membrane is represented by a capacitor and each channel is a combination of a resistance and a voltage source; all channels are connected in parallel. The model is purely empirical and the parameters are chosen so that the results match the data. Here we adopted a ventricular model published by Beeler \& Reuter (BR) [19]. It elaborates on the HodgkinHuxley approach and explicitly involves intracellular calcium and $\mathrm{Ca}^{2+}$ channel. This model captures the AP dynamics of a general mammalian heart on a semi-quantitative level (based on experiments with dog, sheep, calf and cat hearts) and does not provide detailed description of later models, e.g., see Luo \& Rudy [20] or Ten Tusscher et al. [21]. Nonetheless, relative simplicity of the $\mathrm{BR}$ model is preferred in this study. Figure 2 shows schematically four channels involved: sodium $(\mathrm{Na})$, potassium $\left(\mathrm{K}_{1}\right)$, calcium $(\mathrm{Ca})$ and a general channel $\left(\mathrm{X}_{1}\right)$.

The model involves eight variables, the membrane voltage $V_{m}$, concentration of $\mathrm{Ca}^{2+}$ and six other variables describing the states of individual channels:

$$
\frac{d V_{m}}{d t}=-\left(1 / C_{m}\right)\left(i_{K_{1}}+i_{X_{1}}+i_{N a}+i_{s}-i_{\text {external }}\right), \text { (1) }
$$

$$
\begin{aligned}
\frac{[C a]_{i}}{d t} & =-10^{-7} i_{s}+0.07\left(10^{-7}-[C a]_{i}\right), \\
\frac{y_{k}}{d t} & =\left(y_{k}^{\text {inf }}-y_{k}\right) / \tau_{k}, \quad k=1, \ldots, 6,
\end{aligned}
$$

where the current densities through the four channels are

$$
\begin{aligned}
i_{K_{1}}= & 0.35\left\{\frac{4\left(\exp ^{0.04\left(V_{m}+85\right)}-1\right)}{\exp ^{0.08\left(V_{m}+53\right)}+\exp ^{0.04\left(V_{m}+53\right)}}\right. \\
& +\frac{0.2\left(V_{m}+23\right)}{\left.1-\exp ^{-0.04\left(V_{m}+23\right)}\right\}}, \\
i_{X_{1}}= & y_{1} \frac{0.8\left(\exp ^{0.04\left(V_{m}+77\right)}-1\right)}{\exp ^{0.04\left(V_{m}+35\right)}} \\
i_{N a}= & \left(g_{N a} y_{2}^{3} y_{3} y_{4}+g_{N a C}\right)\left(V_{m}-E_{N a}\right), \\
i_{S}= & g_{s} y_{5} y_{6}\left(V_{m}-E_{S}\right), \\
E_{S}= & -82.3-13.0287 \ln \left([C a]_{i}\right)
\end{aligned}
$$

and $i_{\text {external }}$ represents a stimulating current pulse applied for a brief time (1 ms) causing the cell membrane to excite.

The variables $y_{k}$ describe various states of the channels whose dynamics is given by the characteristic times $\tau_{k}$ and resting states $y_{k}^{\text {inf }}$ as follows,

$$
\begin{gathered}
\tau_{k}=1 /\left(\alpha_{k}+\beta_{k}\right), \\
y_{k}^{\inf }=\alpha_{k} /\left(\alpha_{k}+\beta_{k}\right) .
\end{gathered}
$$

Values of all the empirical constants $\alpha_{k}, \beta_{k}$ of the model are found in [19]. Values of channel conductivities $g_{\mathrm{NaC}}$ and $g_{s}$ are 


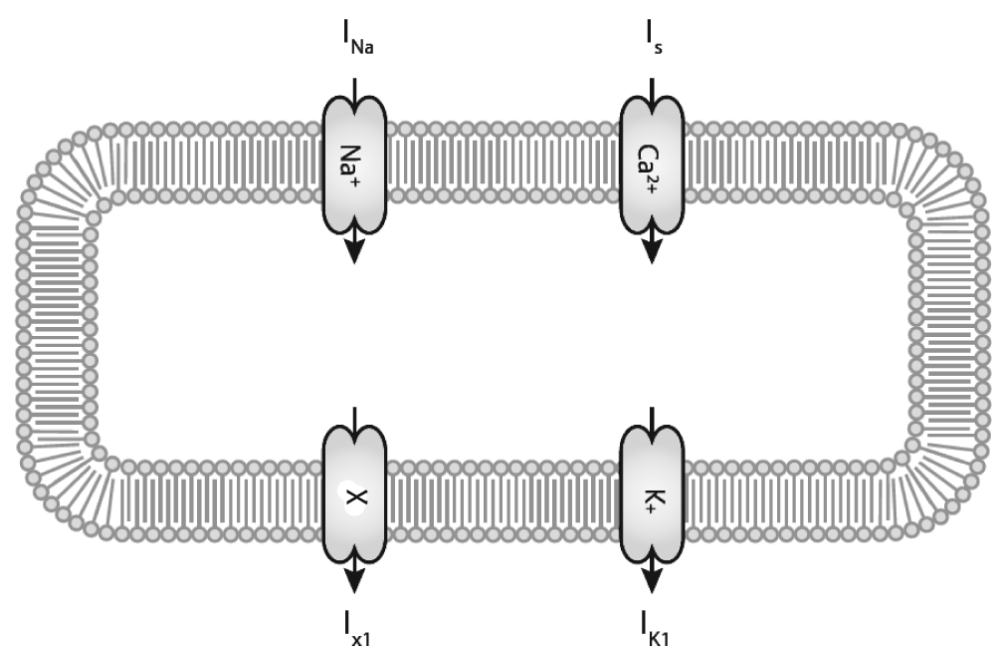

Figure 2 - Schematic representation of the myocardial membrane with four types of channels used in the BR model.

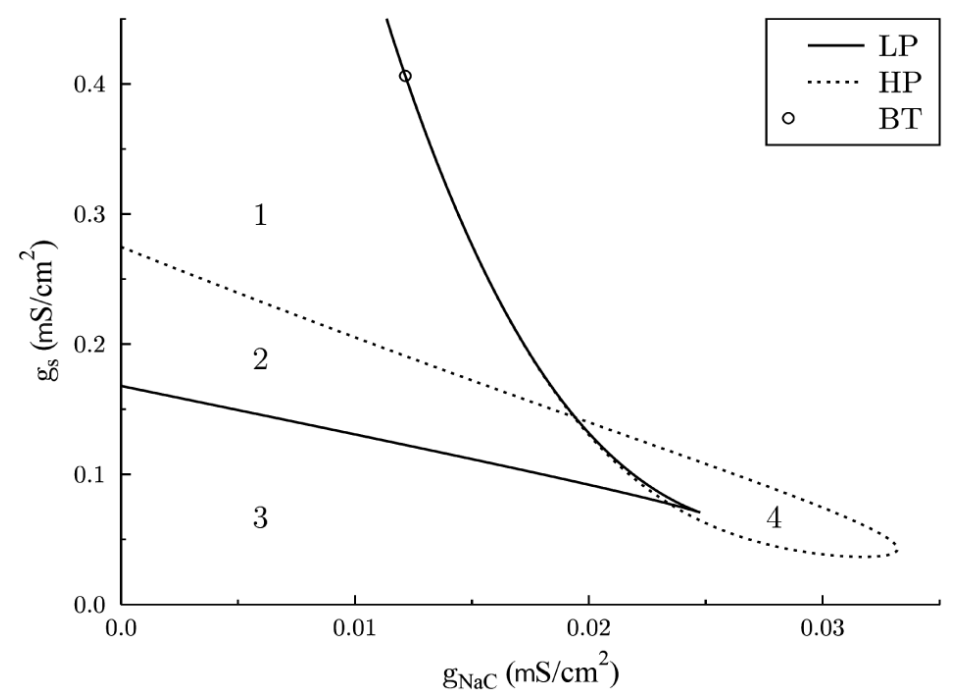

Figure 3 - Bifurcation diagram in the plane of $g_{\mathrm{NaC}}$ and $g_{s}$; region 1 - bistability, regions 2 and 3 excitability, region 4 - periodic oscillations, full line - limit point (or saddle-node) bifurcation, dashed line - Hopf bifurcation, circle - Bogdanov-Takens point (Hopf curve touches the saddle-node curve).

the main adjustable parameters which we use as bifurcation parameters; the values of the other parameters $g_{N a}=4 \mathrm{mS} / \mathrm{cm}^{2}$, $E_{N a}=50 \mathrm{mV}, C_{m}=1 \mu \mathrm{F} / \mathrm{cm}^{2}$ are taken from [19]. Figure 3 shows the parameter plane given by $g_{s}$ and $g_{N a C}$ decomposed into regions with different dynamical modes.

The curves have been obtained by using the program Cont [13] for numerical continuation, stability and bifurcation analysis. Of main interest are the regions 2 and 3 corresponding to excitable dynamics. The figure shows, that the model admits also periodic oscillations (physiologically admissible) and bistability (physiologically irrelevant). For dynamical simulations of networks we chose a point from the excitable region corresponding to $g_{s}=$ $0.09 \mathrm{mS} / \mathrm{cm}^{2}$ and $g_{\mathrm{NaC}}=0.003 \mathrm{mS} / \mathrm{cm}^{2}$. For perturbations we used $i_{\text {external }}=30 \mu \mathrm{A} / \mathrm{cm}^{2}$ for a time duration of $1 \mathrm{~ms}$.

\section{CONNECTIVITY OF THE NETWORK}

As mentioned earlier, cardiac cells interact mainly via gap junctions. We assume here that the AP is spreading quickly along the cell membrane and much more slowly across the GJ, which leads to a discrete-space model, in which each cell is treated as a point object forming a node in the discrete cellular network. An isolated node is described by Eqs. (1)-(9). When coupled into a 
network, coupling terms need to be added. The GJ can be seen as an aqueous pore in which case we can use the Nernst-Planck (NP) equation of electro-diffusion. The cytosol does not flow between cells and so the driving forces are those of concentration difference and potential difference. Assuming that the membrane channels do not carry any charge and assuming isotropic intercellular space between the membranes of the neighboring cells, the potential difference in the NP equation is equal to the voltage difference between membranes. Since the concentration difference of calcium ions is small and the charge transferred between cells is mainly due to potassium ions, which have by far the highest concentration, the coupling term between cells $j$ and $k$ is significantly expressed only in the charge balance, Eq. (1), and the corresponding current density is $i_{G J}=g_{V}\left(V_{m, \text { cell } j}-V_{m, \text { cell } k}\right)$, where $g_{V}$ is the conductivity of the gap junction. This term is widely used in literature, e.g. [7].

In general, Eq. (1) for each cell in a network will have sum of the coupling terms running over all cells that are coupled to the current cell. To conform with the experimental observations of the coupling structure, such as that indicated by Figure 1, the corresponding network is expected to have certain degree of irregularity.

When formulating dynamical equations for such a network, a software tool is needed to generate the explicit expressions for coupling terms in the right-hand sides of the differential equations. For simulations we use the home-made Fortran package Cont [13], which includes the LSODE [22] as a differential equations solver. On the other hand, the freely available software tool NetworkX [11] for formulating and handling complex networks is written in Python. We decided to use the class Graph from the library of NetworkX and write a program heartnet specifying a user-defined class Sit as a subclass of Graph. Sit is an $n$ by $m$ matrix, corresponding to a planar array of cells, in which each node/cell can be connected up to eight neighbors in horizontal, vertical, diagonal and inverse diagonal directions. Despite being only two-dimensional, this approach does respect structure seen in planar preparations of the heart tissue, where the cells are predominantly coupled horizontally, but they are attached to one another also in the vertical direction and because of the branchings (having either $X$ or $Y$ shape) also in two diagonal directions. Couplings are generated randomly but the user can prescribe weights for each of the directions. No-flux and periodic boundary conditions can be generated as well as a number of other features such as assignment of nodes that will be perturbed externally. For example, a network can be generated by running heartnet.

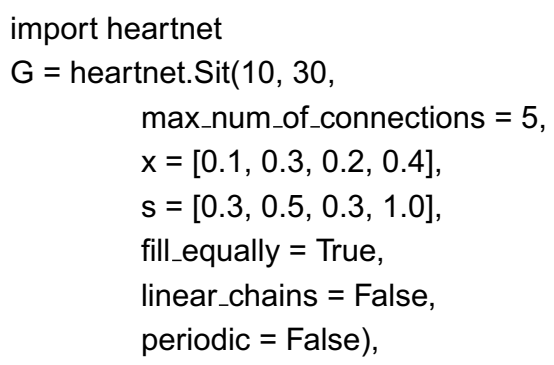

where $\mathrm{G}$ denotes the generated network, which will have 300 nodes in 10 rows and 30 columns, each node will have at most 5 connections with preference on the nodes having the lowest number of connections. The network will have approximately $10 \%$ of nodes in inverse diagonal, $20 \%$ in diagonal, $30 \%$ in vertical and $40 \%$ in horizontal directions. Complete connectivity in horizontal direction (linear chains) is not enforced. Also, weights for coupling strength in all four directions are provided (vector s). Information on various aspects of the underlying graph can be obtained by using functions provided by the library NetworkX. Figure 4 displays the resulting network.

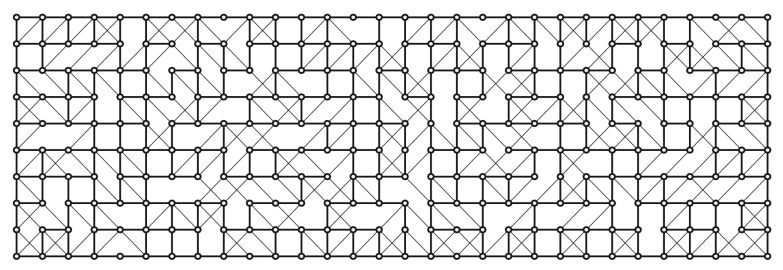

Figure 4 - Schematic of the 10 by 30 network specified in the text.

In addition, another Python program contwrap has been created to generate a Fortran subroutine defining right-hand sides based on the network $G$ (or any other network from the class Graph), a Fortran subroutine defining the initial external stimulation and input data for running the simulation and continuation program Cont [13] (our Python programs as well as Cont are available from the corresponding author). All of these are large files that would be very inconvenient to generate manually.

\section{NETWORK DYNAMICS}

In simulations on two-dimensional continuous-space domains, it is often assumed that the heart tissue is isotropic. Propagating circular waves or rotating spirals were observed, the latter associated with certain arrhythmias. Yet a question remains, how anisotropy and discontinuity of tissue affects the characteristics of the AP spreading. A more realistic view of the cardiac tissue is obtained using the generated network as outlined above. Kanno \& Saffitz [23] examined the connectivity in various parts of the 
cardiac tissue and found that there are four types of couplings between adjacent cells as shown in Figure 5.

They evaluated fractions of each of the connection types in several parts of cardiac tissue. For the left ventricle they provide the following numbers: type a) 29\%, type b) 18\%, type c) $19 \%$, type d) 34\%. Types a) and d) are directly comparable with our vertical and horizontal connectivities, types b) and c) are indistinguishable in our connectivity scheme, thus we consider them jointly and assume that diagonal and inverse diagonal directions are represented equally, that is, to each is assigned $18.5 \%$. In addition, the weights for coupling were taken as fractions of the area available in each direction, namely, 1 for horizontal and vertical directions and 0.375 (average of 0.5 and 0.25 ) for diagonal and inverse diagonal connections.

An example of 50 by 50 network corresponding to the left ventricle is shown in Figure 6.

a)

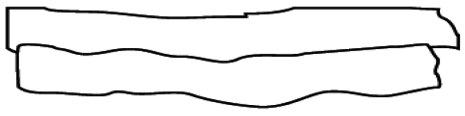

b)

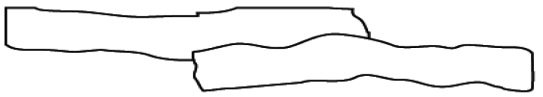

c)

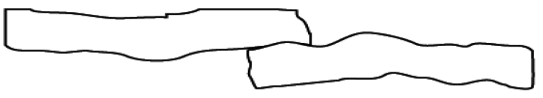

d)

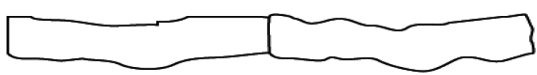

Figure $\mathbf{5}$ - Four type of coupling between myocytes; a) vertical, b) diagonal with large connection area, c) diagonal with small connection area, d) horizontal.

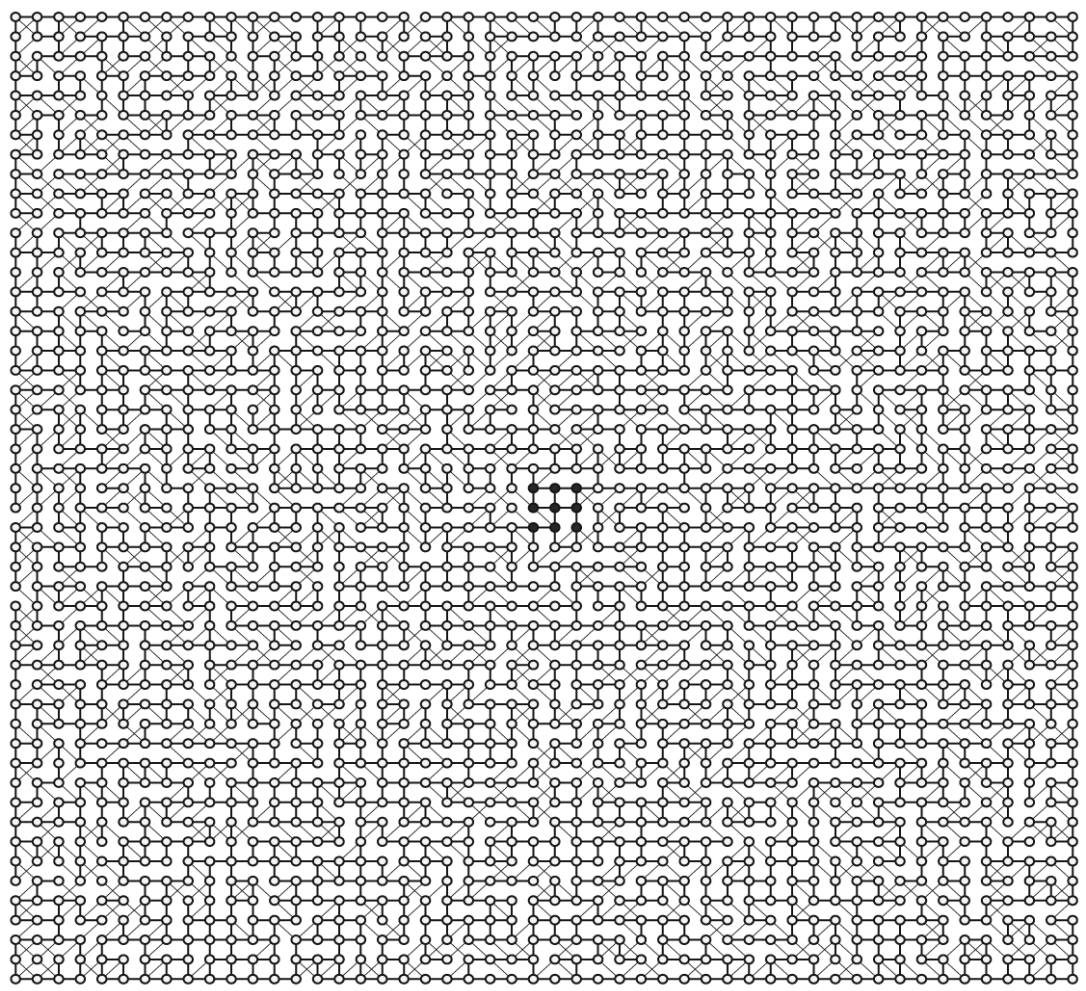

Figure $\mathbf{6}$ - Network used in dynamical simulations of the spreading waves of AP in the left ventricle, full circles denote externally stimulated cells. 
a)

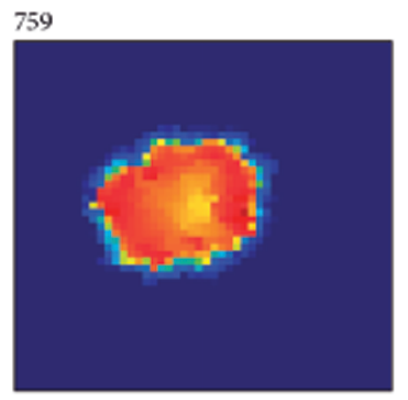

b)

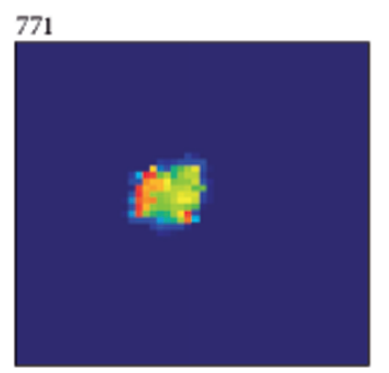

1103

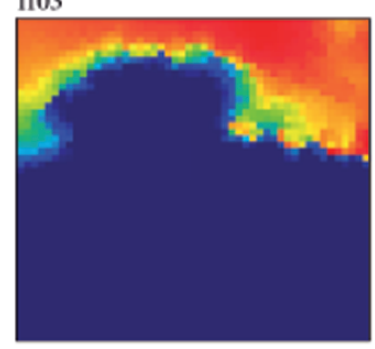

1469

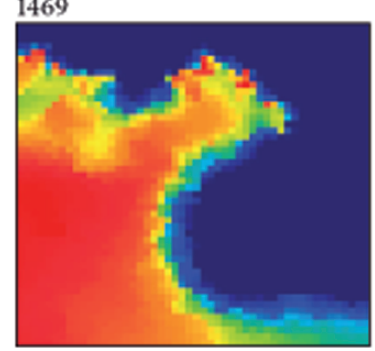

770

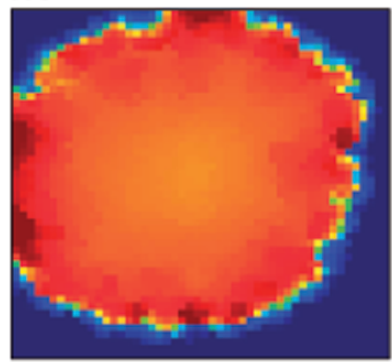

850

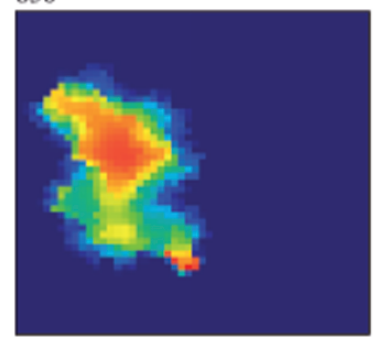

1197

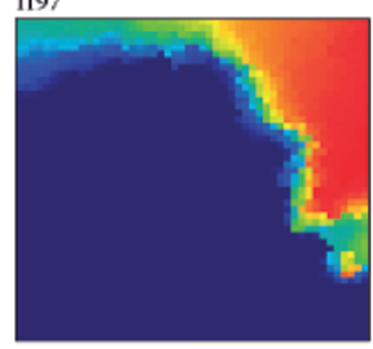

1570

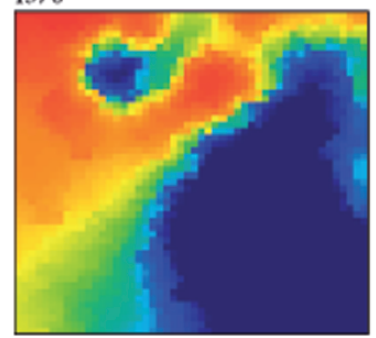

809
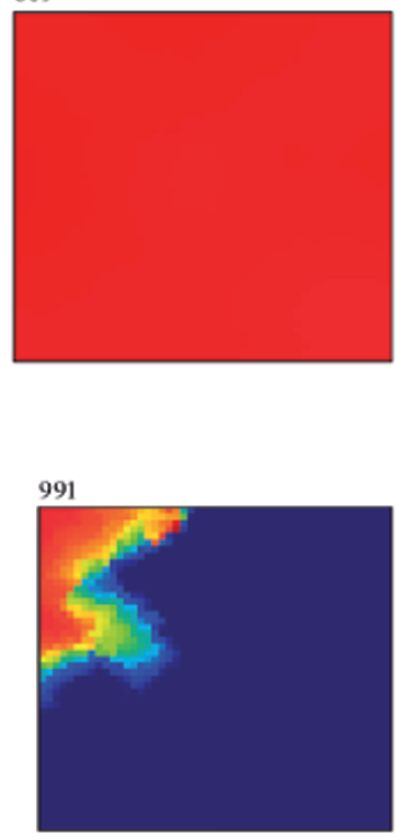

1371

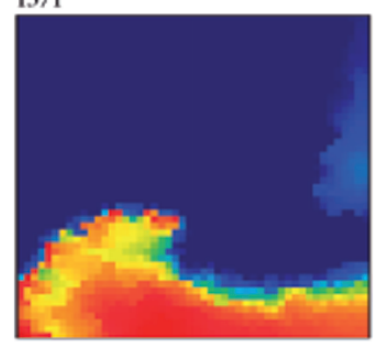

1679

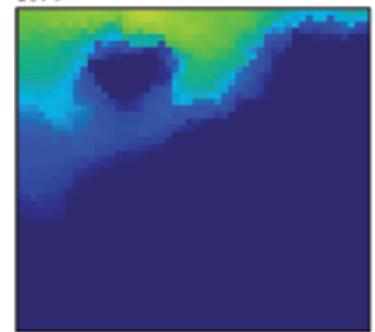

Figure 7 - Depolarization wave stimulated in the middle of the domain of 50 by 50 cells with topology corresponding to the left ventricle; a) $g_{V}=2.1 \mathrm{mS} / \mathrm{cm}^{2}$, b) $g_{V}=0.27 \mathrm{mS} / \mathrm{cm}^{2}$. The number at the top of each panel is time in ms elapsed from the instant of stimulation.

The dynamical simulations using the network in Figure 6 provide a circular, rapidly spreading depolarization wave for a high coupling strength $g_{V}$, see Figure 7a. The wave has rough edges, which is due mainly to anisotropy and to a lesser extent to the discrete lattice. If the coupling strength is decreased, the wave spreads much more slowly and highly irregularly, which again is mainly the effect of anisotropy, see Figure 7b. A similar pattern emerges even when the perturbation is located out of the center and appears also in an array of 100 by 100 cells; thus it is not due to boundary effects. There is a clear tendency toward creating spirals, even though the stimulation creates a transient rather than permanent rotating wave. Nonetheless, this observation points to a significant loss of regularity of the depolarization wave when the gap junctional coupling is weak. 


\section{DISCUSSION AND CONCLUSIONS}

We have shown that anisotropy of cellular networks corresponding to excitable myocardial tissue can have a profound effect on the dynamics of the spreading action potential. This effect is due to locally uneven conditions for signal transmission across the network and is most pronounced when the coupling strength is weak. Such situation may become prominent in an older myocardial tissue since the number of gap junctions is decreasing with age [5]. Tendency toward more complex patterns in a situation of weakened coupling in a heterogeneous array has been observed experimentally in a chicken heart cell culture and simulated by a cellular automaton by Bub et al. [8]. In contrast, Panfilov [7] carried out simulations showing that if one large spiral already exists as a stable dynamical pattern, it can break up into a complex pattern involving many fragments by increasing the coupling strength. The spiral can be recovered from the turbulent pattern by randomly leaving out some intercellular connections. We have not examined stability of a spiral initially existing in a regular cellular system, therefore direct comparison with our results cannot be made. Nonetheless, as pointed out in [7], the mechanism leading to spiral fragmentation at elevated coupling strengths is likely different from the mechanism generating complex spiralling patterns at weak coupling, such as those indicated in Figure 7b. Fishler et al. [6] found in their numerical simulations employing regular arrays with four or six neighbors that dispersion in local coupling strengths implies a planar wave with rough leading edge, but the edge tends to become smooth when the coupling strength in transversal direction is increased. In our case the leading wavefronts do not show smoothening with increased $g_{V}$. This can be explained by different model construction: heterogeneity in the model discussed here is introduced through randomness in the connectivity rather than via variation of local coupling strengths.

The approach presented here has certain limitations, one of them being a rather low number of cells involved in the network and calculations for extended networks are needed. Nonetheless, an increased number of cells is unlikely to alter the main conclusion that anisotropy can potentially be an important factor in explaining occurrence of various types of cardiac arrhythmia. Another limitation is confinement to spatially two-dimensional geometries. However, the method of generating anisotropic networks is easily extendable to three spatial dimensions. The advantage of using Python to produce source code for the dynamical solver is that it is tailor-made and helps in using the computer memory efficiently which in turn is a first step toward effectively solving large-scale networks.

\section{ACKNOWLEDGMENTS}

This work has been supported by the ESF networking programme Funcdyn and by the project of the Czech Science Foundation GAČR 203/09/2091.

\section{REFERENCES}

[1] AIDLEY D. 1998. The physiology of excitable cells. Cambridge University Press.

[2] LECHLEITER J, GIRARD S, PERALTA E \& CLAPHAM D. 1991. Spiral calcium wave propagation and annihilation in Xenopus laevis oocytes. Science, 252: 123-126.

[3] FRIDLYAND LE, TAMARINA N \& PHILIPSON LH. 2010. Bursting and calcium oscillations in pancreatic beta-cells: specific pacemakers for specific mechanisms. Am. J. Physiol. Endocrinol. Metab., 299: E517-E532.

[4] DUPONT G, SWILLENS S, CLAIR C, TORDJMANN T \& COMBETTES L. 2000. Hierarchical organization of calcium signals in hepatocytes: from experiments to models. Biochim. Biophys. Acta., 1498: 134-152.

[5] SCHAPER J, KOSTIN S, HEIN S, ELSASSER A, ARNON E \& ZIMMERMANN R. 2002. Structural remodelling in heart failure. Exp. Clin. Cardiol., 7: 64-68.

[6] FISHLER MG, THAKOR NV \& REID P. 1992. Propagation Wavefronts in Two-Dimensional Topologies of Cardiac Cell Networks. Proceedings of the IEEE Conf. Computers in Cardiology, pp. $411-414$

[7] PANFILOV AV. 2002. Spiral Breakup in an Array of Coupled Cells: The Role of the Intercellular Conductance. Phys. Rev. Lett., 88(11): 118101

[8] BUB G, SHRIER A \& GLASS L. 2002. Spiral Wave Generation in Heterogeneous Excitable Media. Phys. Rev. Lett., 88(5): 058101.

[9] MALATHI R \& REDDY MR. 2007. A Simulation Study on the Effect of Ionic Mechanism Underlying Arrhythmias. IFMBE Proceedings, 14(2): 16-19.

[10] MALATHI R \& REDDY MR. 2009. Effect of Gap Junction Conductance and Formation of Reentry in Human Ventricle Tissue - a Computational Study. Ind. J. of Biomech. (Special Issue NCMB): 85-88.

[11] HAGBERG A, SCHULT D \& SWART P. 2008. Exploring Network Structure, Dynamics, and Function using NetworkX in Proceedings of the $7^{\text {th }}$ Python in Science conference (SciPy), G Varoquaux, T Vaught, J Millman (Eds.), pp. 11-15.

[12] NetworkX. http://networkx.lanl.gov/

[13] KOHOUT M, SCHREIBER I \& KUBIĆCEK M. 2002. A computational tool for nonlinear dynamical and bifurcation analysis of chemical 
engineering problems. Computers \& Chemical Engineering, 26: 517-527.

[14] LOEWENSTEIN WR. 1981. Junctional intercellular communication: the cell-to-cell membrane channel. Physiol. Rev., 61(4): 829-913.

[15] ROHR S. 2004. Role of gap junctions in the propagation of the cardiac action potential. Cardiovasc. Res., 62(2): 309-322.

[16] PERRIARD JC, HIRSCHY A \& EHLER E. 2003. Dilated cardiomyopathy: a disease of the intercalated disc? Trends Cardiovasc. Med., 13(1): 30-38.

[17] KATZ AM. 2011. Physiology of the heart, $5^{\text {th }}$ ed.; Lippincott Williams \& Wilkins; Philadelphia.

[18] HODGKIN AL \& HUXLEY AF. 1952. A quantitative description of membrane current and its application to conduction and excitation in nerve. J. Physiol., 117(4): 500-544.
[19] BEELER GW \& REUTER H. 1977. Reconstruction of the action potential of ventricular myocardial fibres. J. Physiol., 268: 177-210.

[20] LUO CH \& RUDY YA. 1994. A dynamic model of the cardiac ventricular action potential I simulations of ionic currents and concentration changes. Circ. Res., 74: 1071-96.

[21] TEN TUSSCHER KHWJ, NOBLE D, NOBLE PJ \& PANFILOV AV. 2004. A model for human ventricular tissue. Am. J. Physiol. Heart Circ. Physiol., 286: H1573-89.

[22] HINDMARSH AC. 1980. LSODE and LSODI, two new initial value ordinary differential equation solvers. SIGNUM NewsI., 15: 10-11.

[23] KANNO S \& SAFFITZ JE. 2001. The role of myocardial gap junctions in electrical conduction and arrhythmogenesis. Cardiovasc. Pathol., 10(4): 169-177. 